\title{
Experimental study of regular and chaotic transients in a nonsmooth system
}

\author{
Christopher George, Lawrence N. Virgin \\ Department of Mechanical Engineering and Materials Science, Duke University, Durham, \\ NC 27708, USA \\ Thomas Witelski \\ Department of Mathematics, Duke University
}

\begin{abstract}
This paper focuses on thoroughly exploring the finite-time transient behaviors occurring in a periodically driven non-smooth dynamical system. Prior to settling down into a long-term behavior, such as a periodic forced oscillation, or a chaotic attractor, responses may exhibit a variety of transient behaviors involving regular dynamics, co-existing attractors, and super-persistent chaotic transients. A simple and fundamental impacting mechanical system is used to demonstrate generic transient behavior in an experimental setting for a single degree of freedom non-smooth mechanical oscillator. Specifically, we consider a horizontally-driven rigid-arm pendulum system that impacts an inclined rigid barrier. The forcing frequency of the horizontal oscillations is used as a bifurcation parameter. An important feature of this study is the systematic generation of generic experimental initial conditions, allowing a more thorough investigation of basins of attraction when multiple attractors are present. This approach also yields a perspective on some sensitive features associated with grazing bifurcations. In particular, super-persistent chaotic transients lasting much longer than the conventional settling time (associated with linear viscous damping) are characterized and distinguished from regular dynamics for the first time in an experimental mechanical system.
\end{abstract}

Keywords: Impacting, Bi-Stability, Chaotic Transients

\section{Introduction}

Dynamical systems exhibiting discontinuous properties have been a rich area of research [1-16], and the range of possible behaviors in the vicinity of bifurcations is carefully described in [17-19]. Traditional studies tend to focus on steady-state behavior and local bifurcations, and the vast majority of such work has been based on analysis and numerical simulations. This paper carefully examines practical aspects of transient dynamics, and uses an experimental

Preprint submitted to International Journal of Non-Linear Mechanics

November 1, 2015

C) 2015. This manuscript version is made available under the Elsevier user license http://www.elsevier.com/open-access/userlicense/1.0/ 
impacting mechanical system as a testbed to distinguish different classes of behaviors that can occur. We also explore the operational differences in finite time-series data between super-persistent transients and what might conventionally be interpreted as steady oscillations.

Because relatively few experimental studies have been conducted on chaotic transients in nonsmooth systems, this paper will shed light on systems exhibiting grazing bifurcations and super-persistent chaotic transients [20]. A more thorough experimental protocol will be demonstrated to detect the isolated remote attractors, that is, behaviors not ordinarily revealed by the quasi-static continuation (sweeping up/down) of a system with respect to a bifurcation parameter.

Monitoring the behavior of transients induced by small perturbations is the cornerstone of linear stability theory. Here, we extend this notion to include large perturbation-induced transients (more properly termed disturbances) to augment the more familiar bifurcation diagram [21]. Generating an ensemble of initial conditions can be challenging in experimental studies but is shown to be a very valuable element for exploring the global behavior in the system.

\section{Experimental system}

Our experimental system consists of a hard rubber ball (diameter $63 \mathrm{~mm}$, weight $145 \mathrm{~g}$ ) attached to a steel rigid-arm pendulum whose pivot is mounted on a horizontal shake table $[22,23]$. The motion of the pendulum was recorded as time series of the angular position, $\theta(t)$. A rigid wall is placed such that at an angle of $\theta_{\text {wall }}=-30^{\circ}$, the mass at the end of the pendulum arm comes in contact with this fixed barrier. Schematic and photographic images of this system are shown in Figs. 1 and 2 respectively.

The apparatus is attached to a Scotch-yoke forcing mechanism [24] capable of supplying a harmonic excitation to the base over the range of forcing frequencies $\omega_{f}=0.2$ to $2 \mathrm{~Hz}^{1}$ The system was designed so that the natural frequency for resonance of the linearized low-amplitude motion of the pendulum is in this range $\left(\omega_{n} \approx 0.96 \mathrm{~Hz}\right)$. This range gives us access to the broad array of nonlinear large-amplitude behavior involving impacts occurring beyond the fundamental grazing bifurcation, where $\theta=\theta_{\text {wall }}$ is first achieved.

The motion of the pendulum arm is measured to high accuracy using a quadrature encoder. A tachometer is used to extract the instantaneous position of the pendulum at a given forcing phase. The position at a quarter-cycle later, $\tau=1 /\left(4 \omega_{f}\right)$, is also extracted, since subsequent results utilize time-lag embedding, a convenient means of recreating the phase trajectories as well as defining a Poincaré section - an especially useful means of examining dynamic behavior [25].

\footnotetext{
${ }^{1}$ Note that we use Hertz for all frequencies rather than a non-dimensional form.
} 


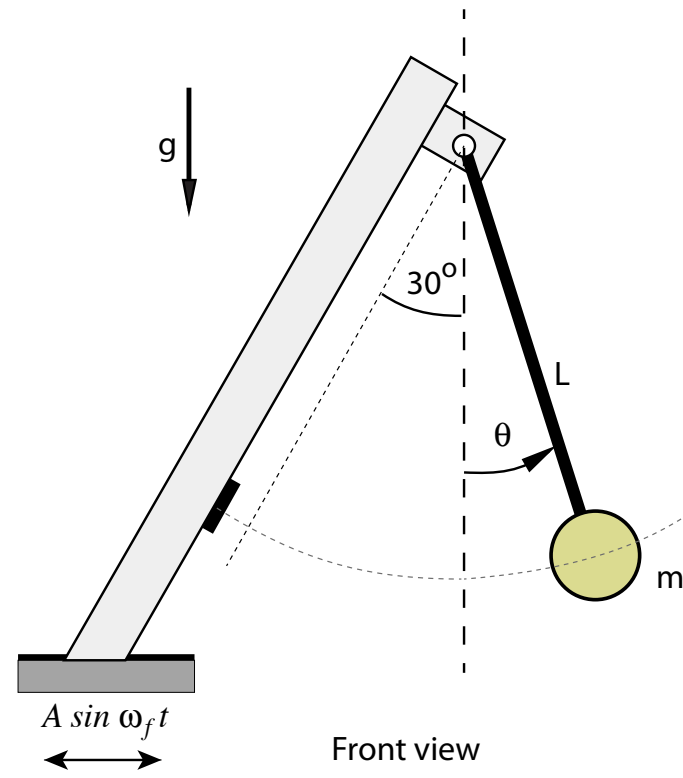

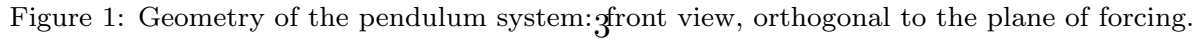




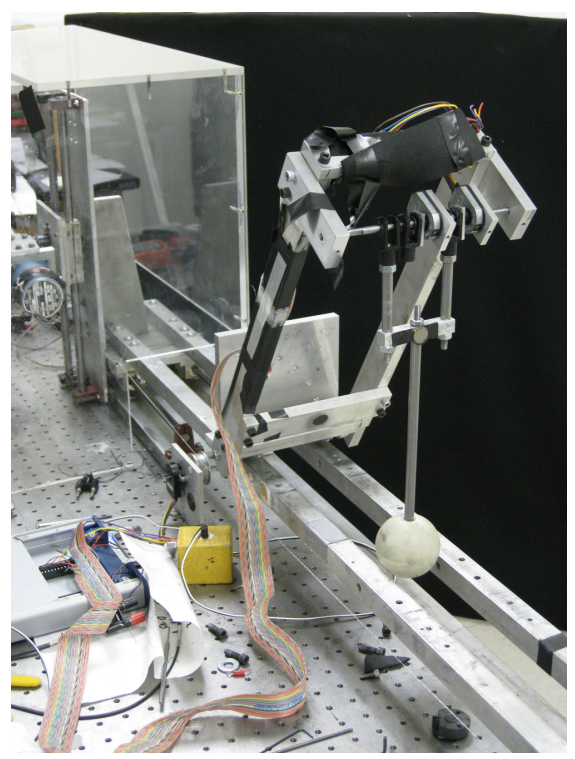

Figure 2: The experimental system. The Scotch-yoke forcing mechanism (rear) drives the motion of the pendulum along parallel rails (foreground). 


\section{Mathematical model}

The system is driven by the imposed horizontal motion of the base, given by $x_{f}(t)=A_{f} \sin \left(\omega_{f} t\right)$, where $A_{f}, \omega_{f}$ are respectively the forcing amplitude and frequency. Then the governing equation of motion for the system can be obtained from Hamilton's principle with an energy dissipation term to model the damping due to friction [23],

$$
\frac{d^{2} \theta}{d t^{2}}+\omega_{n}^{2} \sin (\theta)+\frac{A_{f} \omega_{f}^{2}}{\ell} \sin \left(\omega_{f} t+\phi\right) \cos (\theta)=-D(\theta, \dot{\theta}),
$$

where $\ell$ is the effective length of the pendulum and $\omega_{n}$ is the natural frequency based on this length and the gravitational acceleration, $\omega_{n}=\sqrt{g / \ell}$.

Damping, $D$, in (1a) is modeled by contributions from linear viscous friction and Coulomb friction [26], both assumed to be acting at the pendulum's pivot,

$$
D(\theta, \dot{\theta})=2 \zeta \omega_{n} \dot{\theta}+\mu\left(\dot{\theta}^{2}+\omega_{n}^{2} \cos \theta\right) \operatorname{sgn}(\dot{\theta}),
$$

where $\zeta$ and $\mu$ are friction constants.

Because of the fixed rigid barrier, the motion of the pendulum is limited to $\theta(t) \geq-30^{\circ}$, see Fig. 1. For small $\theta(t)\left(|\theta|<\left|\theta_{\text {wall }}\right|\right)$ there is no impact and the motion is fully determined by (1ab) and will follow the smooth dynamics of a damped driven pendulum. Under forcing yielding $\theta=\theta_{\text {wall }}$, impacts occur, with the minimal case having zero-velocity impacts at grazing bifurcations. Impacts of the pendulum with the wall at time $t\left(\theta(t)=\theta_{\text {wall }}\right)$ are treated as instantaneous inelastic collisions with coefficient of restitution, $0 \leq k<1$, such that the state immediately after impact is given by $\theta\left(t^{+}\right)=\theta_{\text {wall }}$ with

$$
\dot{\theta}\left(t^{+}\right)=-k \dot{\theta}\left(t^{-}\right) .
$$

Values for the system parameters were measured from experimental data and are assumed to be constant with respect to time, see Table 1.

While it is reasonable to question whether the damping parameters might evolve slowly with wear and aging of the system and generation of heat during long experiments, we argue that these variations can be neglected based on the excellent agreement that we are able to obtain with the mathematical model holding these parameters fixed.

Model (1abc) is solved numerically, incorporating a high-resolution (small time-step) capture of the impact condition. Although most of the results reported in this paper are based on experimental data, computed solutions provide verification of the fidelity of the model in reproducing the experimental dynamics, and for some parameter ranges numerics are used to more inten-

sively scrutinize behaviors of special interest. It will be seen that the numerical simulations show excellent agreement with the experimental data.

\section{General behavior}

The forcing frequency provides a convenient control parameter. It is easily changed dynamically in the experimental system and can incremented at a very 
Table 1: System parameters

\begin{tabular}{ll}
\hline Parameter & Description \\
\hline$\ell=26.5 \mathrm{~cm}$ & pendulum arm effective length \\
$\omega_{n}=0.9688 \mathrm{~Hz}$ & linear natural frequency \\
$\zeta=2.1 \times 10^{-2}$ & linear viscous damping ratio \\
$\mu=6 \times 10^{-3}$ & non-dimensional Coulomb damping coefficient \\
$\theta_{\text {wall }}=-30^{\circ}$ & position of the fixed barrier \\
$k=0.557$ & non-dimensional coefficient of restitution \\
$A_{f}=6.35 \mathrm{~cm}$ & non-dimensional amplitude \\
\hline
\end{tabular}

slow rate, and the resulting pendulum response can be extracted as a conventional amplitude, or by displaying the instantaneous position of the pendulum angle at a prescribed forcing phase: the Poincaré section. We shall display both of these views, since the amplitude shows more clearly the response relative to the location of the impact barrier ( at $\theta_{\text {wall }}=-30^{\circ}$ ), and the Poincaré section shows more clearly the periodicity (or lack thereof) of the response. Fig. 3 shows the amplitude response of the system as a function of forcing frequency. The amplitude is displayed in terms of local maxima and minima of $\theta(t)$, i.e., the set of turning points in the time series response. In this way, a chaotic response appears as a rough spread of data, and multi-periodic responses are an even number of point clusters. ${ }^{2}$

An alternative bifurcation diagram, in terms of the Poincaré section for $\theta(t)$ at a prescribed phase, is given in Figure 4, showing a similar comparison for experimental and simulation results. The plot shows instantaneous position of the pendulum at a specific forcing phase, arbitrarily chosen as $\phi=235^{\circ}$, as a different representation of the dynamical state as a function of the forcing frequency $\omega_{f}$.

At each forcing frequency, the experiment was run for two minutes, with only the last 30 seconds plotted, to allow transients to decay out. The color coding is an important feature of this diagram. The black data points were acquired under conditions of very slowly increasing frequency starting from $\omega_{f} \approx 0.3 \mathrm{~Hz}$. The response is periodic and non-contacting until about $\omega_{f} \approx 0.8 \mathrm{~Hz}$ when contact with the barrier first occurs and the system immediately transitions into a short burst of chaos before rapidly turning into a reverse period-doubling cascade ending in a period-two motion at $\omega_{f} \approx 0.83 \mathrm{~Hz}$. This, finally bifurcates to a period-one impacting state at $\omega_{f} \approx 0.94 \mathrm{~Hz}$, which eventually disappears as the system returns to a non-impacting response at $\omega_{f} \approx 1.42 \mathrm{~Hz}$.

The orange data points were collected from a slowly decreasing frequency sweep starting from a non-impacting oscillatory state at $\omega_{f}=2 \mathrm{~Hz}$. While

\footnotetext{
${ }^{2}$ In this, and many of the figures, the order in which colors are plotted yields an unavoidable visual artifact for overlapping points, thus in some places the black data points are obscured by the orange points.
} 


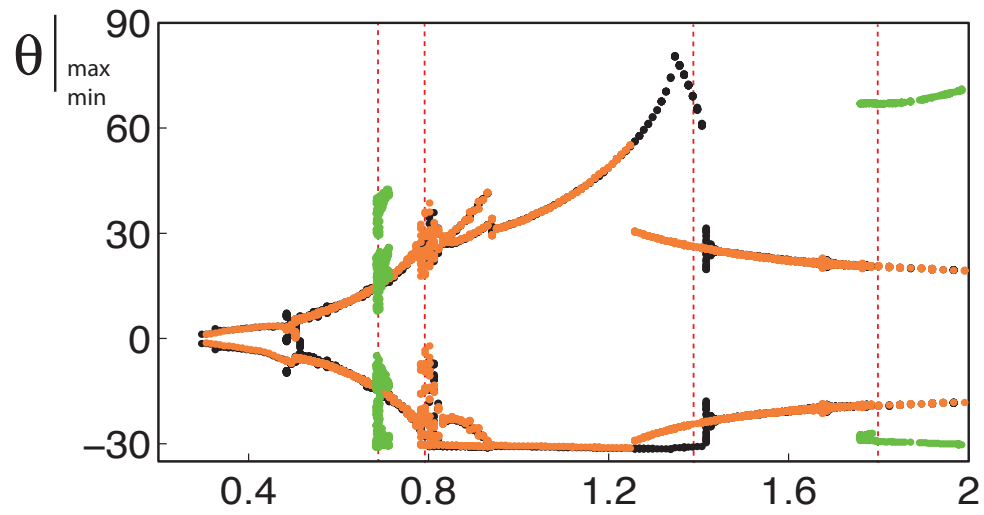

(a)

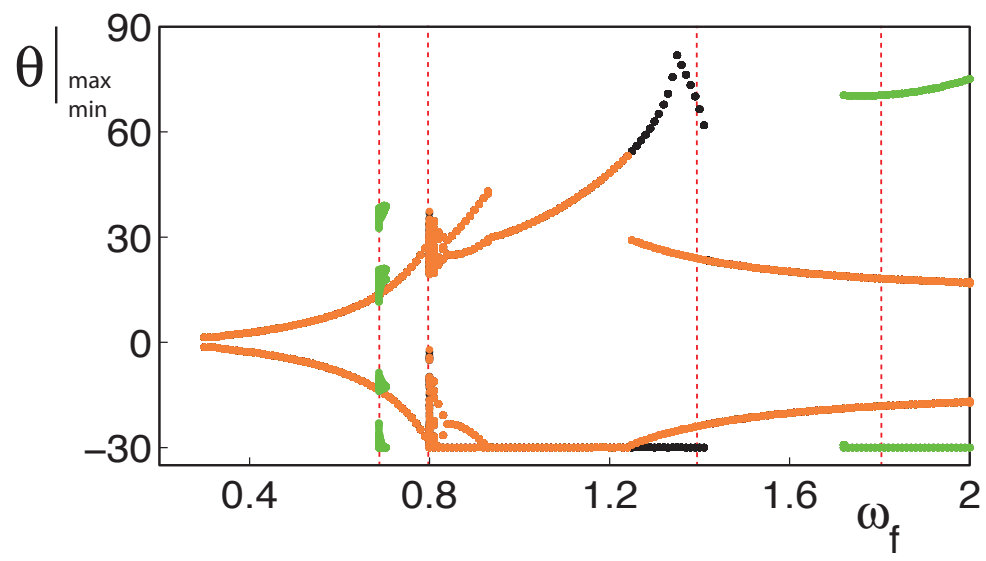

(b)

Figure 3: Bifurcation diagram: Maxima and mninima of the response angle $\theta(t)$ (in degrees) versus forcing frequency (in Hertz), (a) experimental data, (b) numerical simulation of model (1abc). The black data points correspond to a sweep-up in frequency, the orange to a sweepdown, and green points are remote attractors. The vertical lines indicate forcing frequencies, $\omega_{f}=\{0.69,0.795,1.39,1.81\} \mathrm{Hz}$, where the system will be studied in detail in later sections and Figure 5. 


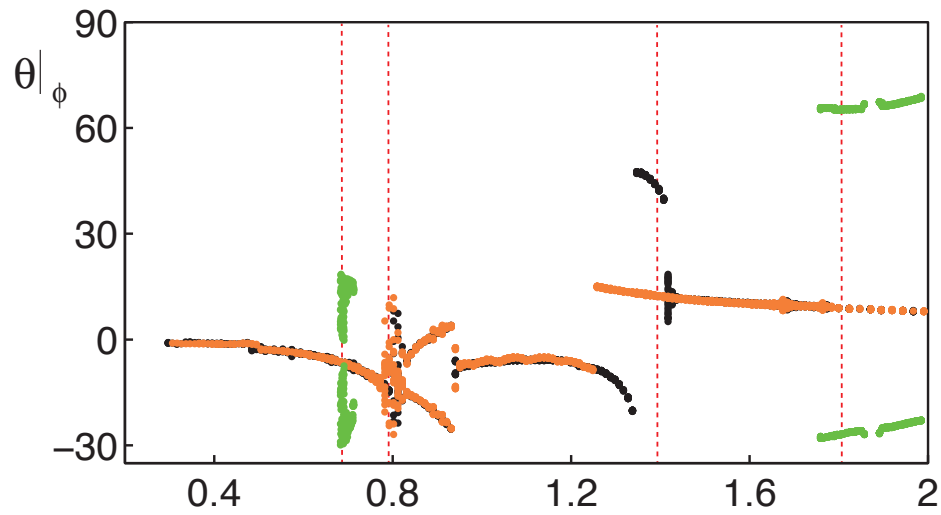

(a)

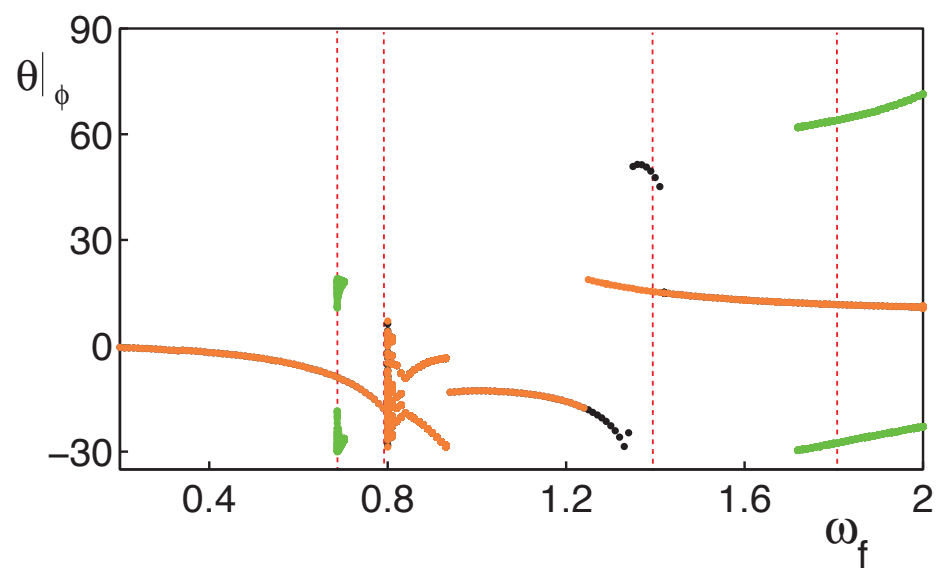

(b)

Figure 4: Bifurcation diagram: Poincaré sectiog for $\theta$ (in degrees) versus forcing frequency (in Hertz), (a) experimental data, (b) numerical simulation. The black data points correspond to a sweep-up in frequency, the orange to a sweep-down, and green points are remote attractors. 
exactly overlapping with the sweep-up over most of the diagram, the most obvious difference is that the decreasing frequency sweep follows the non-impacting response to a lower frequency $\left(\omega_{f} \approx 1.25 \mathrm{~Hz}\right)$, meaning that the system has a sizable frequency range with coexisting attractors, one impacting and one non-impacting, on $\omega_{f} \approx 1.25-1.42 \mathrm{~Hz}$.

An additional item to note in Fig. 3 and Fig. 4 is that there are two sets of green data points in each bifurcation diagram. These are remote attractors; steady-state behaviors that are not captured in the sweep up or sweep down, and are found through disturbances or more generic choices of initial conditions. To find them experimentally, the system was set to the middle of the frequency range, perturbed until the remote attractor was found, and then swept up until the attractor disappeared. This was then repeated, but with a sweep down to find the lower limit of the attractor. (Note that if the sweep down does not begin at exactly the same spot as the sweep up, a gap can appear in the data, which occurs at $\omega_{f}=1.84 \mathrm{~Hz}$ in the experimental bifurcation, giving the false impression of two separate attractors.). It is also worth noting that the green data in the vicinity of $\omega_{f} \approx 1.9 \mathrm{~Hz}$ was discovered experimentally and then confirmed numerically, while the opposite was true for the green data in the vicinity of $\omega_{f} \approx 0.7 \mathrm{~Hz}$. This provides strong validation of the mathematical model and associated estimation of the system parameters.

The different behaviors identified in the production of Fig. 3 and 4 are now subjected to close experimental scrutiny. A sample of time series and time-lag projections of some of these behaviors is shown in Fig. 5. A property of this experimental system is that in each range where there are two attractors, the current state can be quickly distinguished by the presence or absence of impacts. In later plots, points colored red will be used to indicate the impacting response, and blue points for the non-impacting state. The properties of the system at the three forcing frequencies from Fig. 5, and at the fundamental grazing bifurcation at $\omega_{f} \approx 0.8 \mathrm{~Hz}$ will be examined in the following sections.

\section{Non-impacting vs. period-one impacting states}

The first frequency range explored is $\omega_{f} \approx 1.25-1.42 \mathrm{~Hz}$, where there are two coexisting responses. These responses show the typical behavior of a bi-stable system; the sweep up follows the impacting branch until it goes unstable around $\omega_{f} \approx 1.42 \mathrm{~Hz}$, and a sweep down from there follows the non-impacting branch until it disappears at $\omega_{f} \approx 1.25 \mathrm{~Hz}$. These coexisting attractors are reminiscent of hysteretic behavior in a smooth dynamical system.

This is a well-understood phenomena, and is one of the characteristic behaviors in the vicinity of grazing [18]. To look at the relative dominance of the two attractors, and to see how their transients behave, data for an experimental basin of attraction was collected. To find this data, a procedure to generate initial conditions was designed, referred to as the Random Disturbance Procedure (RDP). To begin, forcing is started and the system is followed through its decay onto the steady state. Each Poincare section it passes through is a valid initial condition for whatever attractor it ends up on. Once a predefined 

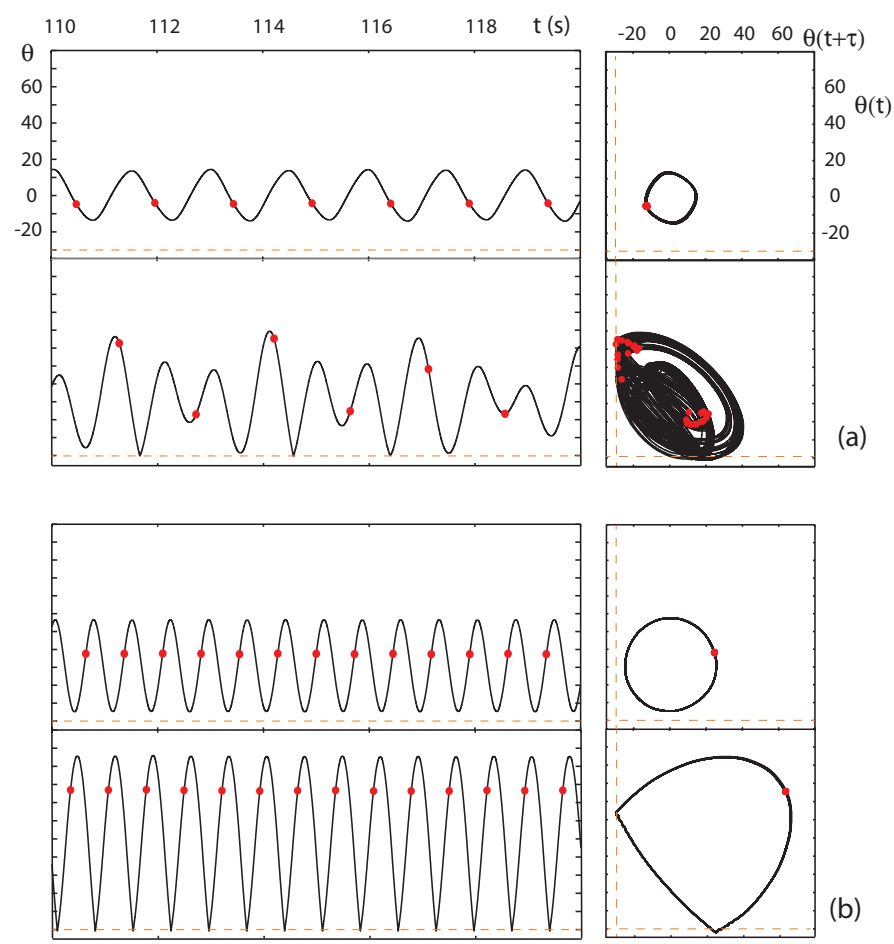

(b)
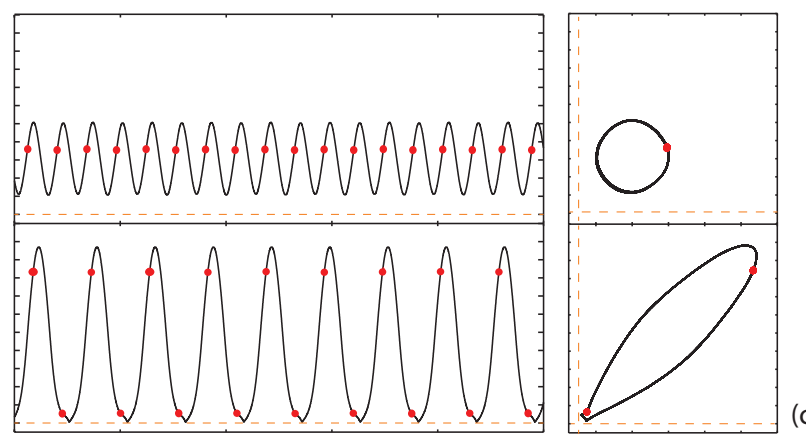

(c)

Figure 5: Gallery of dynamic behaviors. Each set shows two coexisting responses, impacting and non-impacting at: (a) $\omega_{f}=0.69 \mathrm{~Hz}$ described in Sec. 7 (b) $\omega_{f}=1.39 \mathrm{~Hz}$ described in Sec. 5 (c) $\omega_{f}=1.81 \mathrm{~Hz}$ described in Sec. 6. Time series for $\theta(t)$ are shown in the left column, the right column is the corresponding time-lag projection. Red dots are the Poincaré section. Note that these time-delay plots swap horizontal and vertical axes from the conventional layout so that the Poincaré section lines up horizontally between the time-lag and time series plots. 
time has elapsed, the system is disturbed in some manner (for this system, applying a different forcing frequency for a short period of time was a convenient disturbance method) and then returned to the target frequency. Once again the system can decay to steady state (though due to the disturbance this may be a different steady state than the previous), generating a new set of initial conditions. With enough repetitions, this process generates an experimental analogue of an initial condition/basin of attraction plot. This method is also sometimes referred to as stochastic interrogation $[27,28]$.

It is important to note that this transient behavior can be displayed in the phase plane (position versus velocity), or using a time-delay plot (position at the Poincare section phase versus position at a time $\tau=1 /\left(4 \omega_{f}\right)$ (a quarter of a forcing cycle) later). Time-delay plots will be primarily used here, as velocity is not measured directly, and numerical differentiation of experimental data is problematic in terms of amplifying measurement noise. While a time-delay plot can be topologically equivalent to a phase plane diagram for smooth dynamical systems, there are some special issues that arise due to the impacts in the system, as discussed in Sec. 7 [29].

The RDP was performed for 200 disturbances at $\omega_{f}=1.39 \mathrm{~Hz}$, and the resulting basins of attraction are shown in Fig. 6. This plot shows that the impacting state dominates the non-impacting state over a majority of the space. In fact, only four trials out of the 200 led to the non-impacting (blue) attractor, and hence this can be considered a rare attractor. The transients in the local vicinity of the non-impacting steady-state have been used to estimate damping [24]. It is interesting that while this forcing frequency is very near the disappearance of the impacting state in a saddle-node bifurcation occurring at $\omega_{f}=1.42 \mathrm{~Hz}$ (as described in [18]), its basin of attraction dominates.

\section{Non-impacting vs. period-two impacting states}

Over the range of $\omega_{f} \approx 1.7-2.0 \mathrm{~Hz}$ there are two coexisting attractors, but unlike the two attractors described in Sec. 5, here, the attractor for the impacting state was not found in the sweep up or the sweep down. As it is a remote attractor, it can be studied using the RDP as in [21].

Fig. 7 shows that in this range, the impacting behavior (red) is period two. This can be seen in the bifurcation diagram in Fig. 4 in the two branches of the remote attractor (green) on the Poincaré section at $\theta=-30^{\circ}$ and $\theta \approx 70^{\circ}$.

\section{Non-impacting vs. chaotic impacting states}

Below grazing $\left(\omega_{f}<0.8 \mathrm{~Hz}\right)$, the system exhibits a somewhat surprising remote attractor. For about $\omega_{f} \approx 0.68-0.74 \mathrm{~Hz}$, there is a chaotic attractor, which again was not detected during sweeping up or sweeping down. The expectation would be that since this frequency is below grazing and below the natural frequency of the linearized pendulum, only small-amplitude responses would be observed. However, this is not the case. The random disturbance procedure was again used to map the geometry of this attractor's basin of attraction. 


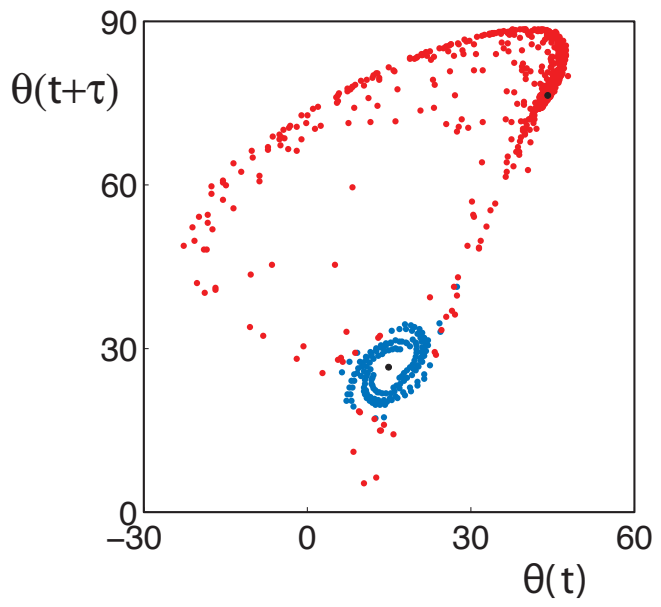

Figure 6: Experimental data showing the basins of attraction at $\omega_{f}=1.39 \mathrm{~Hz}$. Red points approach the period-one impacting state, blue points approach the non-impacting state. 


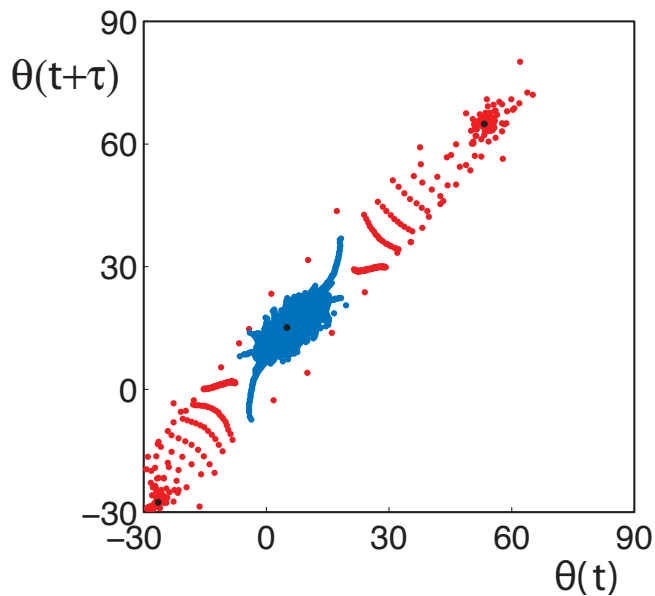

Figure 7: Experimental data showing the basins of attraction at $\omega_{f}=1.81 \mathrm{~Hz}$. Red points approach the period-two impacting state, blue points go to the non-impacting state. 


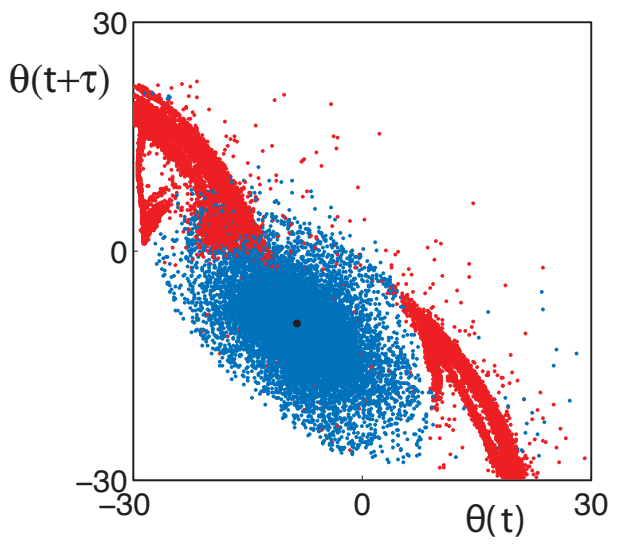

Figure 8: Experimental data showing the baqins of attraction at $\omega_{f}=0.69 \mathrm{~Hz}$. Red points approach the chaotic impacting state, blue points go to the non-impacting state. 
Fig. 8 shows the result of the random disturbance procedure with red being the impacting chaotic response, and blue the periodic non-impacting response. What is particularly interesting is the region around $(-15,5)$. This region seems to show a mixing of red and blue, and an overlapping of basins of attraction.

For a smooth three-dimensional dynamical system, this cannot occur as the Poincaré section in time-delay coordinates is two-dimensional; the basins should be distinct in two dimensions [30]. However, the topological equivalence between delay plots and phase portraits is dependent on the system being smooth, and the non-smooth event can cause a folding of the delay space. The nature of this folding can be observed by using a second delay [29].

The three-dimensional basin is shown in Fig. 9. While it is difficult to convey the structure of this three-dimensional object in two-dimensional plots, two view angles give the reader an idea of the geometry. A majority of the blue points lie on a tilted plane, while most of the red points fold up from this plane. It is important to observe that in two dimensions the system appears to have a mix of red and blue, but in three dimensions the two colored regions are distinct.

To give a different representation of the basins of attraction in two dimensions, we compute the phase plane numerically for trajectories starting from initial conditions at $t=0,(\theta(0), \dot{\theta}(0))$. Using the mathematical model, it is simple to set a uniform grid of initial conditions and then determine the color by whether the solution converges to the impacting or non-impacting state. The resulting basin of attraction, shown in Fig. 10(a), has well-defined borders and a clear structure. In part (b) this is also represented in terms of a time-lag embedding plot. We indeed observe the apparent overlapping of the basins as in Fig. 9. Furthermore, although the trajectories were initiated on a regular grid, they must pass through the Poincaré section associated with the delay, and hence are subject to strong distortion of the phase space.

\section{Chaotic transients}

Fig. 11 shows the detailed structure of the bifurcation diagram in the neighborhood of the grazing bifurcation near $\omega_{f}=0.8 \mathrm{~Hz}$. This data set was collected to more closely observe the behavior before and after grazing, as well as to find a more precise value for the exact frequency at which the grazing bifurcation occurs. On initial inspection, it is reasonable to believe that in the neighborhood of $\omega_{f}=0.8 \mathrm{~Hz}$ there are once again two coexisting attractors, as the transition between chaos and periodicity is slightly different in a sweep up versus a sweep down (see Fig. 11(a)). It appears to be similar to the behavior seen in Sec. 7 . However, crucially, when these sweeps are repeated allowing a longer time for transients to decay, i.e. 5 minutes as opposed to 1 minute, the system appears to have a smaller region of chaos than previously observed, as shown in Fig. 11(b).

Given an arbitrary initial condition, a typical trajectory will undergo some transient motion prior to settling onto its attractor. In a conventional linear system, this is often expressed as a settling time, $T_{s}$. The estimate from linear theory for a settling time for a damped driven linear oscillator to approach the 


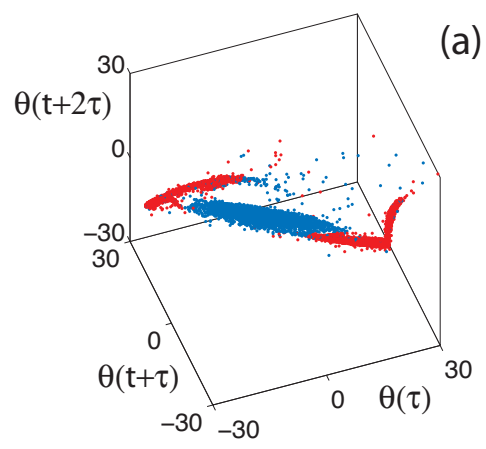

(a)

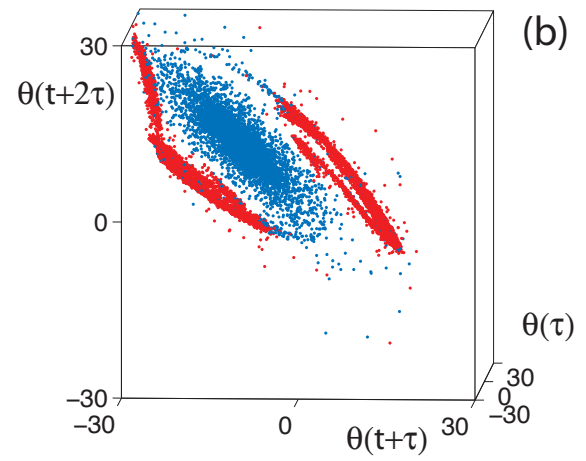

Figure 9: Experimental data showing the basins of attraction at $\omega_{f}=0.69 \mathrm{~Hz}$ extending the Poincaré section using two delays, $\tau$ and $2 \tau$. Two viewing angles are shown of this threedimensional plot to help show the exact shape. Red points approach the chaotic impacting state, blue points approach the periodic non-impacting state. 


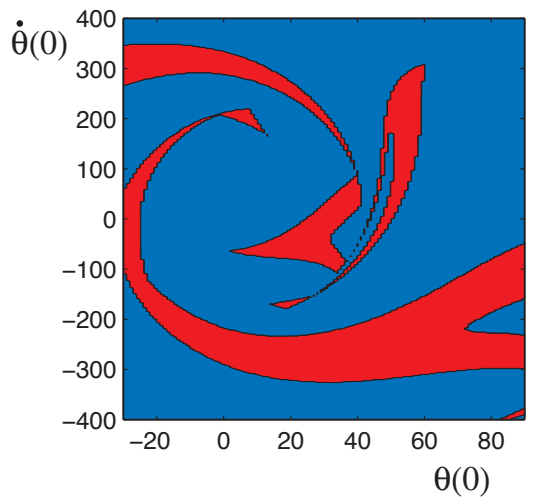

(a)

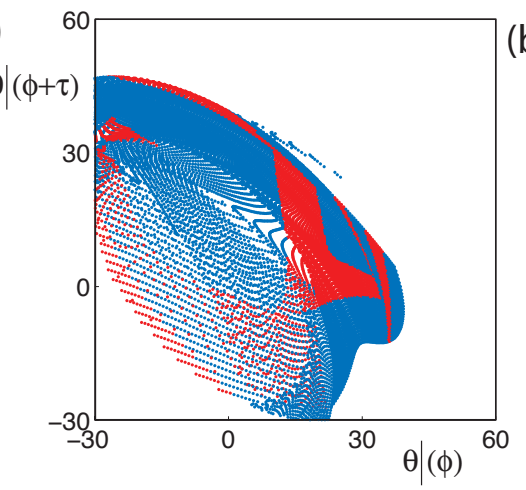

(b)

Figure 10: Numerical simulations of the basins gf $^{f}$ attraction at $\omega_{f}=0.695 \mathrm{~Hz}$, (a) in the initial condition phase plane, and (b) in time-lag coordinates. Red indicates the chaotic impacting state, blue is the non-impacting state. 


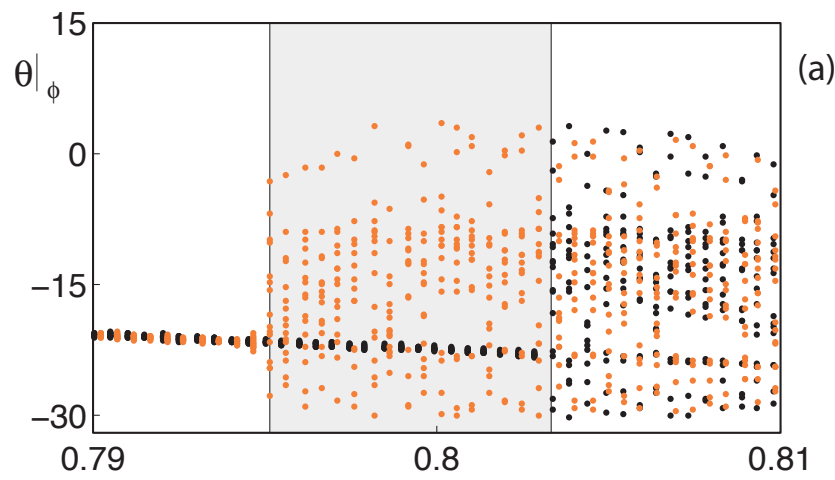

(a)

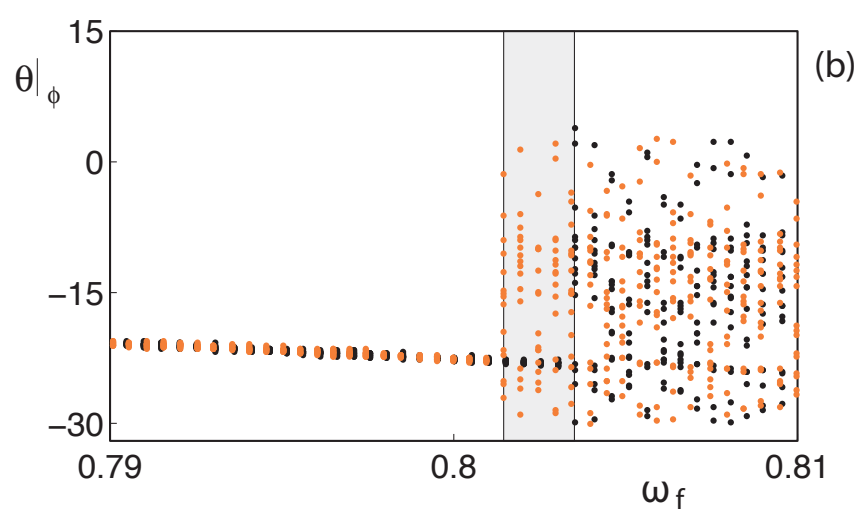

Figure 11: Experimental data showing the Poincaré section bifurcation diagram in the vicinity of grazing, $0.79 \leq \omega_{f} \leq 0.81 \mathrm{~Hz}$ : (a) using fast sweeps (1 minute for transients), (b) using slow sweeps ( 5 minutes for transients). The gray-shaded areas indicate frequency ranges with apparent coexisting attractors. 
final response within $2 \%$ is $T_{s} \approx 4 /\left(\zeta \omega_{n}\right)$ [31]. For the $\zeta$ and $\omega_{n}$ given in table 1 , the settling time for the system in question is $313 \mathrm{sec}$, or approximately 5 minutes. Thus, conventionally, it would appear reasonable to use 5 minutes as an appropriate length of time for transients to decay, and consider the system to be close to steady state.

However, close investigation of the system in the vicinity of grazing shows that this time is not sufficiently long for some ranges of forcing frequencies. Fig. 12 shows two typical time series and corresponding phase projections (using time-lag embedding). Starting from an arbitrary set of initial conditions, the motion shown in $(a, b)$, undergoes a few cycles of transient behavior then appears to settle into chaos (red Poincaré points) but subsequently converges onto the periodic attractor (as indicated by the blue Poincaré points) after about a minute or so. Starting from different initial conditions, parts (c) and (d) show a similar situation but where the final transition to chaos occurs after about 8 minutes or so. The phase trajectories correspond to behavior over the range of $4<t<6$ minutes. The disappearance of these chaotic responses given a long enough time frame suggests that rather than a chaotic attractor, these responses are actually super-persistent chaotic transients [20]. Super-persistent chaotic transients have been observed in electrical systems [32] and have been shown to be possible in aeroelastic systems [33], but this is the first example of these long-lived transients demonstrated in a single degree of freedom nonsmooth mechanical experiment.

While early behavior of the time series from Fig. 12a at $\omega_{f}=0.795 \mathrm{~Hz}$ is consistent with the chaos observed with fast sweeps in Fig. 11a, it might have have been thought that Fig. 11b suggests that chaotic transients of 5 minutes or longer do not exist at that forcing frequency. However in the following subsections it will be shown that these two observed behaviors are not at odds with each other since the lengths of chaotic transients will have distributions of times over all trajectories.

\subsection{Finite-time basins of attraction}

Since these chaotic responses disappear given long enough time, the periodic state is the global attractor in this range of forcing frequencies. However, it is interesting to treat the chaotic transients as an effective attractor and generate basins of attraction for finite times, when some chaotic transients have decayed out, and others have not. These will be referred to as finite-time basins of attraction, and the coloring will be determined by whether or not an impact has occurred within the last 10 forcing cycles before the next disturbance in the RDP.

Fig. 13 shows two finite-time basins of attraction extracted from the experimental data at $\omega_{f}=0.795 \mathrm{~Hz}$. The data corresponds to many induced transients that are tracked and classified according to which behavior they exhibit at the end of the run. Plot (a) shows the trajectories color-coded based on the behavior two minutes after the initial conditions. We see a relatively small area of light blue - the basin of attraction corresponding to the periodic (non-impacting) transient behavior. The other, larger area of red data points 

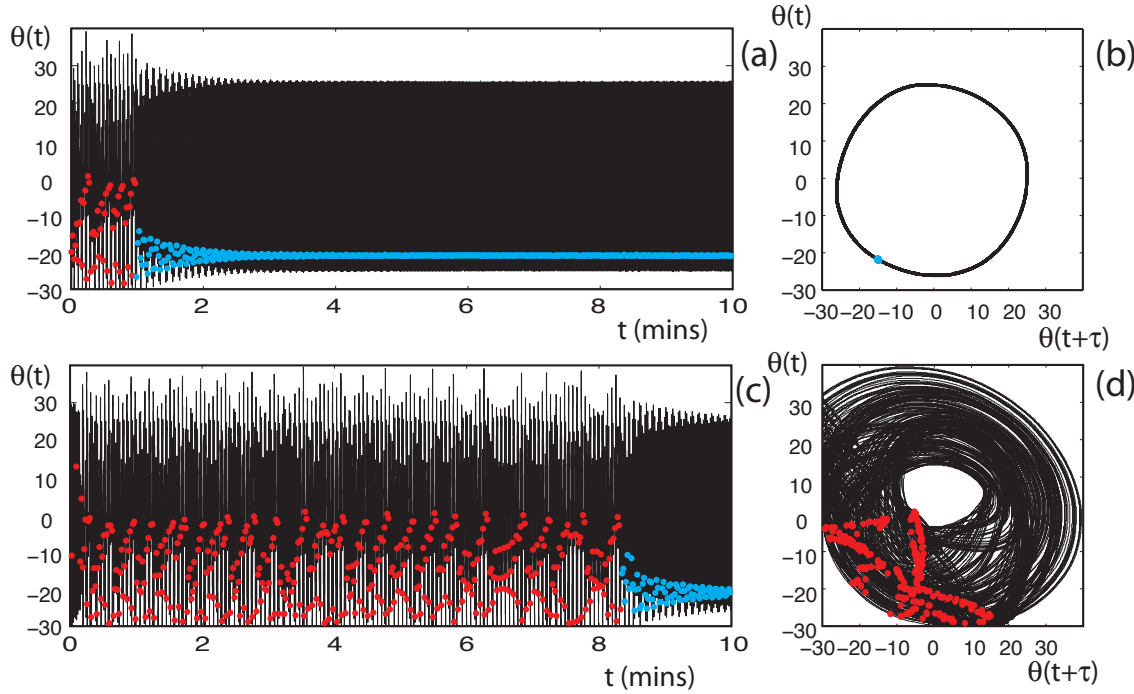

Figure 12: $(\mathrm{a}, \mathrm{c})$ Two experimental time series runs at $\omega_{f}=0.795 \mathrm{~Hz}$. Starting from different initial disturbances, each run begins with chaotic/impacting behaviors and transitions to periodic behavior at some time. Red and Blue dots mark the impacting/non-impacting Poincaré section points. (b,d) Corresponding time-lag plots for the time series over times $4<t<6$ min showing apparent convergence to attracting states. 
are those in which the behavior is essentially chaotic (typically involving an impact every three forcing cycles). The presence of a few blue data points within the red suggests that a small number of transitions (from chaotic to periodic) has already taken place. After 15 minutes most of the formerly red points have now becomes blue (part b). This process continues until all the data turns blue - chaos has disappeared. Especially in the shorter time scale, the finitetime basin of attraction appears similar to a riddled basin in that the initial conditions for the two attractors are mixed without pattern or division between basins. Riddled basins are a behavior associated with the onset of chaos [34, 35].

This speckled structure of the finite-time basin appears similar to the effect shown near the point $(-15,5)$ in Fig. 8. One can imagine that representing Fig. 13(a) in three dimensions might separate the blue from the red points that appear mixed together in the current projection. Fig. 14 shows that with an additional delay coordinate, the basins of attraction resides on a curved surface that only appears to overlap itself when reduced to two dimensions. However, it is distinctly different than the 3-dimensional delay shown in Fig. 9 in that while the attractor is no longer folded over itself, the blue and red points are still intermixed. This indicates that there is a different behavior occurring here than at $\omega_{f}=0.69 \mathrm{~Hz}$.

Another interesting aspect of chaotic transients is the fact that they do not have a monotonic decay towards steady state. In contrast, linear transients tend to have an exponential decay envelope, which will manifest in the phase portrait as swirls or rays moving toward the attractor. This gives an observer the ability to measure how close the transient is to reaching the steady state, and also see where the steady state is located. Chaotic transients for this system, however, tend to fall onto a fractal geometry, and dwell there for a seemingly arbitrary length of time with no obvious trend towards the final attractor [36]. The shape of this intermediate stage can be shown by plotting the chaotic transients up until the last impact, having skipped the first few forcing cycles to let the system settle onto the chaotic transient finite-time attractor. This is shown in Fig. 15 for $\omega_{f}=0.795 \mathrm{~Hz}$. This figure should be compared with Fig. 16 which is the system at a slightly higher forcing frequency $\left(\omega_{f}=0.810 \mathrm{~Hz}\right)$, where chaos is the sole attractor. The chaotic transient attractor bears a resemblance to the folded, fingered chaotic attractor at the slightly higher forcing frequency.

The chaotic transients are associated with a boundary crisis at grazing [36]. This is in some ways reminiscent of the ghosting behavior more commonly seen in the vicinity of a saddle-node bifurcation (but before it has occurred), with the transients dwelling very close to the future location of the node for extended periods of time [30, 37].

\subsection{Length of chaotic transients}

As shown in Fig. 12, these chaotic transients can last for dramatically different lengths of time. An alternative way of studying the structure of Fig. 13 is to consider the time of last impact for each run. Separating out runs that were non-impacting for all times, we can construct a histogram for the fraction of runs 

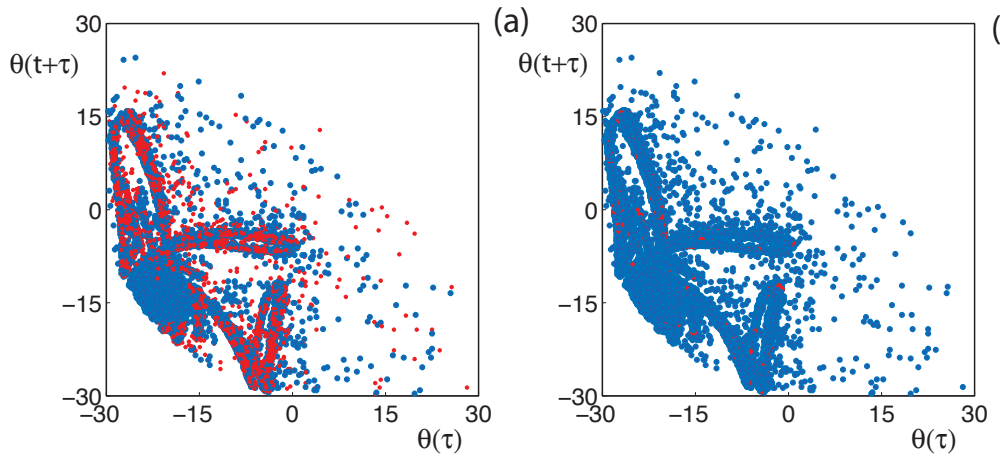

(b)

Figure 13: Transition of the distribution of states in the finite-time basins of attraction at $\omega_{f}=0.795 \mathrm{~Hz}$ (red - chaotic, blue - periodic): (a) after 2 minutes, (b) after 15 minutes. 

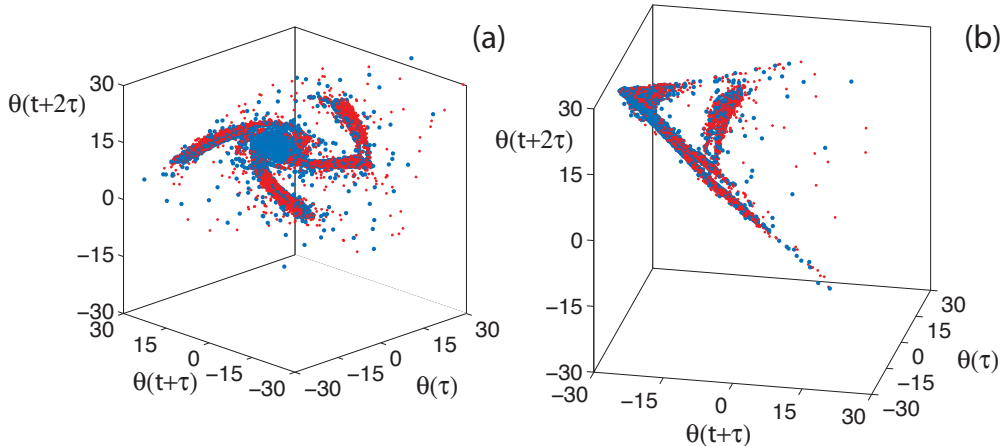

Figure 14: Experimental data showing the finite-time basins of attraction at $\omega_{f}=0.795 \mathrm{~Hz}$ after two minutes, using two delays in addition to the Poincaré section. As the resulting plot is 3 dimensional, two viewing angles are shown to help show the full structure. Red points indicate runs that are still impacting after 2 minutes, while blue points show runs where impacting ended before two minutes elapsed. 


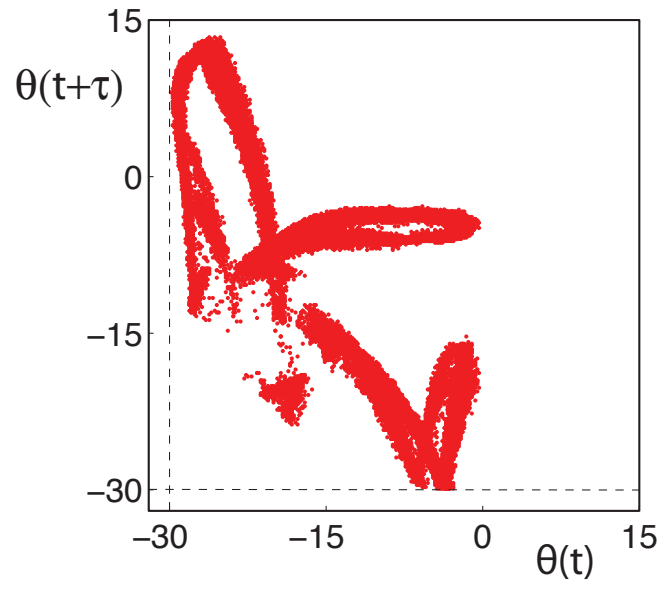

Figure 15: The finite-time chaotic attractor, with the first 20 forcing cycles omitted, at $\omega_{f}=$ $0.795 \mathrm{~Hz}$. 


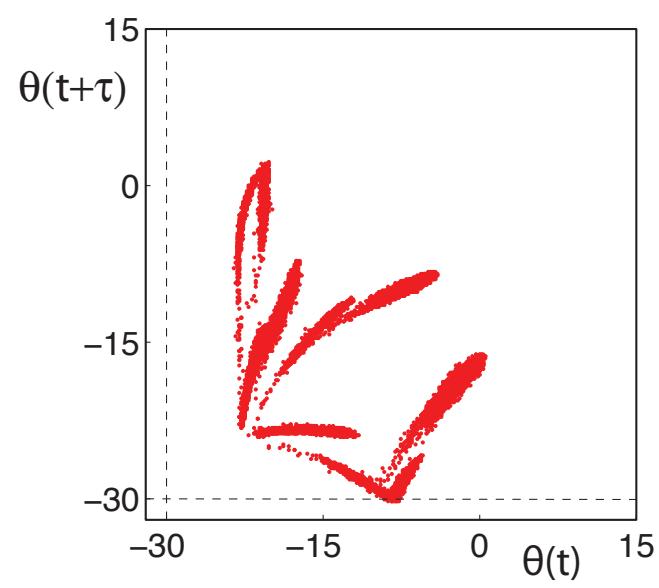

Figure 16: The chaotic attractor at $\omega_{f}=0.810 \mathrm{~Hz}$. 
whose final impact occurred at a given time. We call this measure $\delta(t)$, which gives the fraction of trajectories whose chaotic (impacting) behavior persists to time $t$. Fig. 17 shows that $\delta(t)$ can be represented by exponential distributions, $\delta(t) \approx e^{-\gamma t}$. This exponential profile is characteristic of super-persistent transients [20], and gives a sense of how the finite-time basin of attraction (and chaotic transients) changes with time.

The rate of this decay varies with forcing frequency, as can be seen for the three frequencies (below or close to the grazing bifurcation at $\omega_{f}=0.803 \mathrm{~Hz}$ ) tested experimentally in Fig. 17. Each distribution was calculated from 200 runs of 20 minutes each, which involved over two days of experimental collection per forcing frequency. To look at additional frequencies, numerical simulations of the mathematical model was used. Results from the numerical simulations' persistence of chaos is shown in Fig. 18.

It is convenient to characterize these varying decay rates is by fitting a exponential of the form $\delta(t) \approx e^{-\gamma t}$ where $\gamma\left(\omega_{f}\right)$ is the rate of decay characterizing the distribution of chaotic transient lengths. Fig. 19 shows $\gamma\left(\omega_{f}\right)$ for a range of forcing frequencies near grazing, obtained from the numerical simulation data shown in Fig. 18. Note that for the range near $\omega_{f}=0.68 \mathrm{~Hz}$ where a co-existing chaotic state occurs, only runs settling down to the periodic non-impacting state were used for Fig. 19. This shows a trend of lower $\gamma$-and therefore longer transients-as the system approaches the grazing bifurcation. As this represents an exponential timescale, it is useful to see how this compares to the timescale of the viscous damping. For a linear viscous system, the transients decay on the scale of $e^{-\omega_{n} \zeta t}$. This decay rate $\left(\gamma=\omega_{n} \zeta\right)$ has been added as a green line in Fig. 19. When the black curve falls below this, it indicates that the timescale for transient decay is longer than the timescale for linear viscous decay, and these chaotic transients are likely to be extremely long lasting.

Indeed, Fig. 19 shows that approaching the transition to persistent chaos (at grazing), the system's chaotic transients grow longer. Part (a) shows a wide range of forcing frequencies, most of which have higher decay rates and hence shorter timescales for the lengths of chaotic transients. Part (b) shows the vicinity of grazing at $\omega_{f}=0.803 \mathrm{~Hz}$ where super-persistent transients can occur. The green line represents the timescale for the viscous damping. Note that above $\omega_{f}=0.803 \mathrm{~Hz}$ the line ends, as grazing has occurred, and all trajectories remain impacting $(\gamma=0)$.

\section{Concluding Remarks}

Based on a relatively high fidelity experimental study (validated by numerical simulations) this paper has highlighted the following interesting features found in the transient dynamics of a non-smooth dynamical system:

- A regime of hysteresis delineating co-existing periodic responses. The relative dominance of the attractors may not follow the more familiar hysteric behavior found in smooth systems (i.e. near the disappearance of the impacting state, the impacting state may still be dominant). 


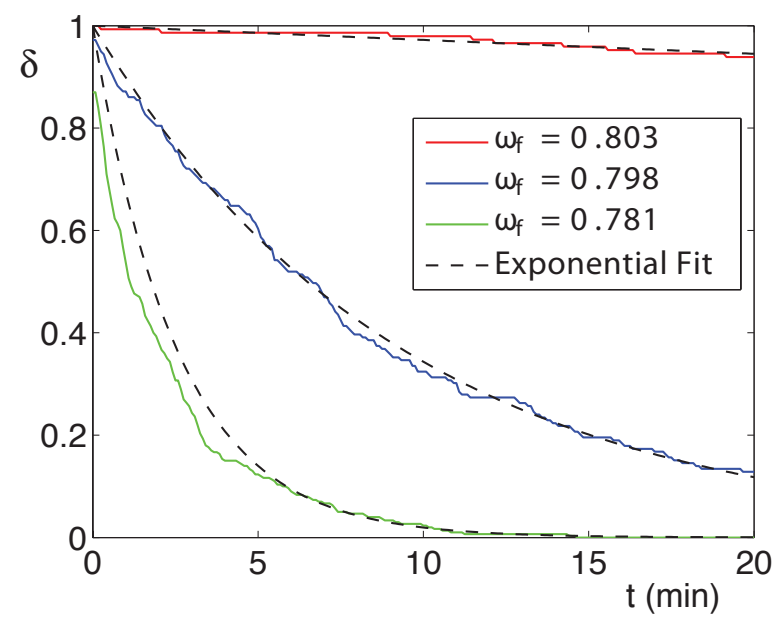

Figure 17: Experimental data showing the transition from chaos to periodicity at three forcing frequencies. The dashed black lines show exponential fits to the distribution of the final-impact times in large ensembles of experimental trajectories generated by the RDP. 


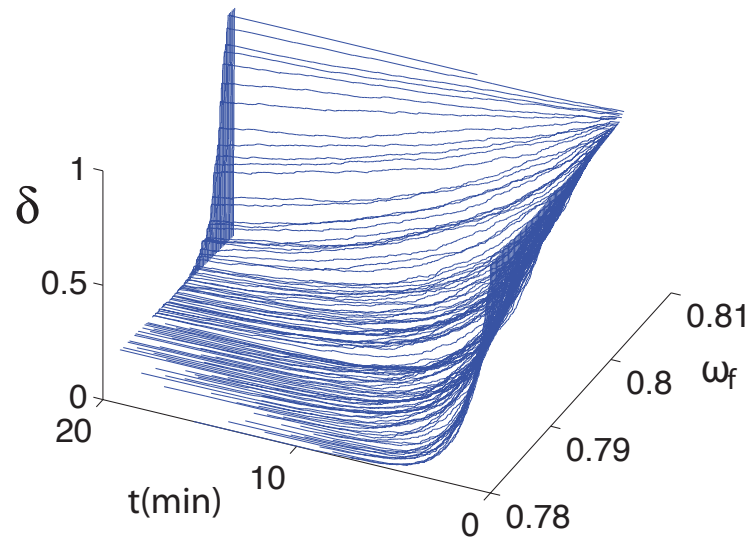

Figure 18: The transition from chaos to periodicity in numerical simulations for a range of forcing frequencies. 


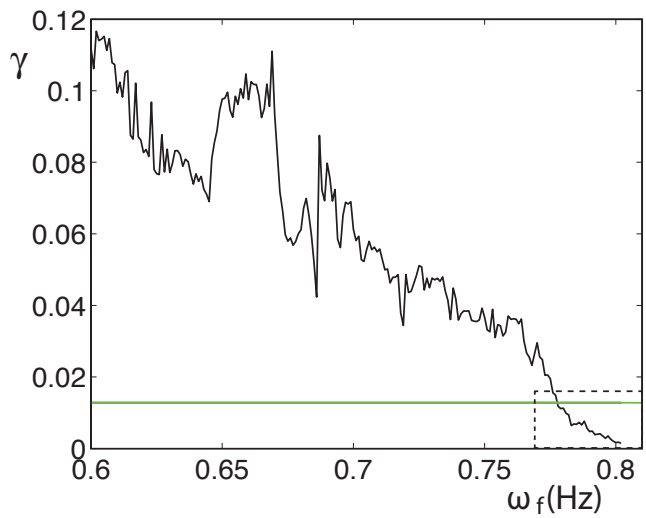

(a)

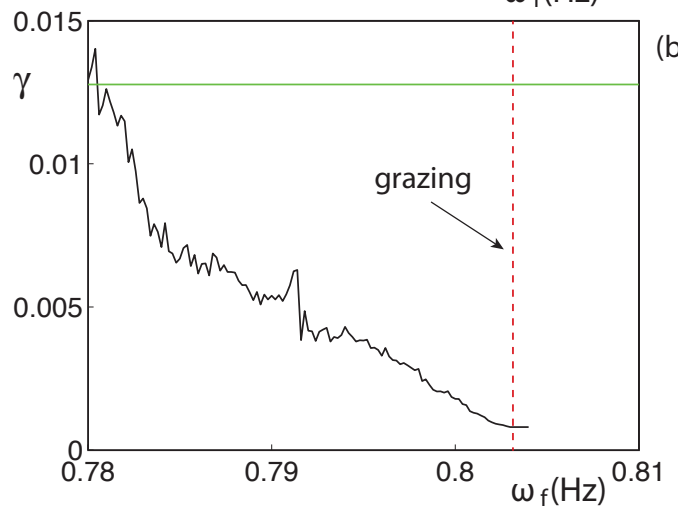

(b)

Figure 19: Exponential decay rate for chaotic transients as a function of $\omega_{f}$ : (a) over a wide range of forcing frequencies, compared to the decay rate set by viscous damping (green line), and (b) near grazing, showing super-persistent transients. 
- Even after completing a sweep up and a sweep down, there can be additional attractors, unconnected to the main branches, which can exhibit chaotic, or very large amplitude responses. These isolated responses may have relatively small basins of attraction, and in a practical sense locating all such behaviors can represent a daunting task.

- A grazing event that transitions directly to chaos may exhibit superpersistent chaotic transients just below the point of grazing, which gives the appearance of hysteresis in a sweep up and sweep down, depending on the rate of sweeping.

- Depending on the exact nature of the system, these super-persistent transients may last long enough that they need to be considered robust finitetime responses in their own right, since for many real-world applications periods of time like twenty minutes may span very large numbers of operating cycles.

- Given the practical differences between impacting and non-impacting responses, e.g., in terms of predicting the useful lifetime of a system subject to mechanical fatigue, the ability to better characterize persistent transient behaviors seems a very important direction for further studies.

Acknowledgement: The support of the Dynamical Systems Program of the National Science Foundation (grant number 0927186) is gratefully acknowledged.

\section{References}

[1] P. J. Holmes, The dynamics of repeated impacts with a sinusoidally vibrating table, Journal of Sound and Vibration 84 (2) (1982) 173-189.

[2] S. W. Shaw, P. Holmes, Periodically forced linear oscillator with impacts: Chaos and long-period motions, Physical Review Letters 51 (8) (1983) 623-626.

[3] J. M. T. Thompson, R. Ghaffari, Chaotic dynamics of an impact oscillator, Physical Review A 27 (3) (1983) 1741-1743.

[4] C. N. Bapat, S. Sankar, N. Popplewell, Repeated impacts on a sinusoidally vibrating table reappraised, Journal of Sound and Vibration 108 (1) (1986) 99-115.

[5] R. M. Everson, Chaotic dynamics of a bouncing ball, Physica D 19 (1986) $355-383$.

[6] A. B. Nordmark, Non-periodic motion caused by grazing incidence in an impact oscillator, Journal of Sound and Vibration 145 (2) (1991) 279-297.

[7] F. Peterka, J. Vacik, Transition to chaotic motion in mechanical systems with impacts, Journal of Sound and Vibration 154 (1) (1992) 95-115. 
[8] G. S. Whiston, Singularities in vibro-impact dynamics, Journal of Sound and Vibration 152 (3) (1992) 427-460.

[9] C. Budd, F. Dux, Intermittency in impact oscillators close to resonance, Nonlinearity 7 (1994) 1191-1224.

[10] H. E. Nusse, E. Ott, J. A. Yorke, Border-collision bifurcations: An explanation for observed bifurcation phenomena, Physical Review E 49 (2) (1994) 1073-1076.

[11] A. P. Ivanov, Bifurcations in impact systems, Chaos, Solitons \& Fractals 7 (10) (1996) 1615-1634.

[12] M. D. Todd, L. N. Virgin, An experimental impact oscillator, Chaos, Solitons \& Fractals 8 (1997) 699-714.

[13] L. N. Virgin, C. J. Begley, Grazing bifurcations and basins of attraction in an impact-friction oscillator, Physica D 130 (1999) 43-57.

[14] K. Popp, Non-smooth mechanical systems, Journal of Applied Mathematics and Mechanics 64 (5) (2000) 765-772.

[15] P. Thota, X. Zhao, H. Dankowicz, Co-dimension-two grazing bifurcations in single-degree-of-freedom impact oscillators, Journal of Computational and Nonlinear Dynamics 1 (2006) 328-335.

[16] M. di Bernardo, C. J. Budd, A. R. Champney, P. Kowalczyk, A. B. Nordmark, G. O. Tost, P. T. Piiroinen, Bifurcations in nonsmooth dynamical systems, SIAM Review 50 (4) (2008) 629-701.

[17] W. Chin, E. Ott, H. E. Nusse, C. Grebogi, Grazing bifurcations in impact oscillators, Physical Review E 50 (6) (1994) 4427-4444.

[18] W. Chin, E. Ott, H. E. Nusse, C. Grebogi, Universal behavior of impact oscillators near grazing incidence, Physical Letters A 201 (1995) 197-204.

[19] F. Casas, W. Chin, C. Grebogi, E. Ott, Universal grazing bifurcations in impact oscillators, Physical Review E 53 (1) (1996) 134-139.

[20] C. Grebogi, E. Ott, J. A. Yorke, Super persistent chaotic transients, Ergodic Theory and Dynamical Systems 5 (1985) 341-372.

[21] J. Waite, L. N. Virgin, R. Wiebe, Competing responses in a discrete mechanical system, International Journal of Bifurcation and Chaos 24 (2014) $1430003-1$.

[22] P. V. Bayly, L. N. Virgin, An experimental study of an impacting pendulum, Journal of Sound and Vibration 164 (2) (1993) 364-374.

[23] T. Witelski, L. N. Virgin, C. George, A driven system of impacting pendulums: Experiments and simulations, Journal of Sound and Vibration 333 (6) (2014) 1734-1753. 
[24] L. N. Virgin, Introduction to Experimental Nonlinear Dynamics, Cambridge University Press, 2000.

[25] H. Kantz, T. Schreiber, Nonlinear time series analysis, Cambridge, 1997.

[26] B. F. Feeny, A nonsmooth coulomb friction oscillator, Physica D 59 (1992) $25-38$.

[27] J. R. Cusumano, B. W. Kimble, A stochastic interrogation method for experimental measurements of global dynamics and basin evolution: Application to a two-well oscillator, Nonlinear Dynamics 8 (1995) 213-235.

[28] K. D. Murphy, P. V. Bayly, L. N. Virgin, J. A. Gottwald, Measuring the stability of periodic attractors using perturbation-induced transients: Applications to two non-linear oscillators, Journal of Sound and Vibration 172 (1) (1994) $85-102$.

[29] B. F. Feeny, J. W. Liang, Phase-space reconstructions and stick-slip, Nonlinear Dynamics 13 (1997) 39-57.

[30] S. H. Strogatz, Nonlinear Dynamics and Chaos, Westview Press, 1994.

[31] W. J. Palm, Modeling, Analysis and Control of Dynamic Systems, John Wiley \& Sons, 1983.

[32] L. Zhu, A. Raghu, Y.-C. Lai, Experimental observation of superpersistent chaotic transients, Physical Review Letters 86 (2001) 4017-4020.

[33] H. Dai, X. Yue, D. Xie, S. N. Atluri, Chaos and chaotic transients in an aeroelastic system, Journal of Sound and Vibration 333 (26) (2014) 7267 7285 .

[34] J. C. Alexander, J. A. Yorke, Z. You, Riddled basins, International Journal of Bifurcation and Chaos 2 (4) (1992) 795-813.

[35] E. Ott, J. C. Alexander, I. Kan, J. C. Sommerer, J. A. Yorke, The transition to chaotic attractors with riddled basins, Physica D 76 (4) (1994) 384-410.

[36] C. Grebogi, E. Ott, J. A. Yorke, Fractal basin boundaries, long-lived chaotic transients, and unstable-unstable pair bifurcation, Physical Review Letters 50 (1983) 935-938.

[37] S. T. Trickey, L. N. Virgin, Bottlenecking phenomenon near a saddle-node remnant in a duffing oscillator, Physics Letters A 248 (1998) 185-190. 